\title{
Introduction
}

\section{THE ROLE OF ARGUMENTATION}

The best way to learn syntax is not simply to study it, but to do it. The purpose of this book is to bring readers to the point where they can "do syntax" themselves. This ability is essential for understanding the field and reading its burgeoning literature.

We have designed the book for students in linguistics, for those in related fields, and for those studying linguistics for what it can contribute to their general education. We have found that focusing on syntactic argumentation is the key to meeting the needs of each of these groups.

The student who learns how to use linguistic data to argue for one hypothesis over another learns the essence of scientific method. An important advantage of linguistics in this respect is that its data is generally much more accessible than data in other sciences and typically can be obtained without timeconsuming experiments. In the course of constructing syntactic arguments, the student discovers that each time a set of data leads to the rejection of one hypothesis, another must be formulated and tested against further data. In this way, one is led to investigate language in greater and greater depth and to discover the surprising intricacy of what may initially have seemed to be a familiar and ordinary phenomenon.

Syntactic argumentation is also crucial for the student who wishes to go further in linguistics. For this student, it is as important to learn the reasons for a theory as it is to learn the theory itself. Particular theories and proposals will give way to others in time. What remains most stable are the standards of argumentation and the criteria for choosing among competing hypotheses. In 
addition, as new theories replace old, each new one is expected to account for the data covered by its predecessors. Thus, in constructing arguments the student not only learns why some hypotheses have been rejected in favor of others, but also becomes familiar with data that has shaped the direction of the field.

Most arguments in syntax use data to show that if one makes certain assumptions, then certain conclusions follow. Examples of various argument forms are the following:

(1) Given assumptions $A, B$, and $C$, the data requires one to conclude $D$ as well.

(2) Given $A, B$, and $C$, the data requires one to reject $D$ in favor of $E$.

(3) Given $A, B$, and $C$, the data is compatible with either $D$ or $E$.

(4) The data makes it impossible to maintain $A, B$, and Cjointly; at least one of them must be given up.

In general, arguments are not absolute. They typically do not show that something must be the case, independent of other assumptions. Rather, they show that given specific assumptions, certain conclusions follow.

In some cases the arguments we present are quite abstract. For example, after giving an argument of the form (1), we might speak of "freeing conclusion D from assumption B." Doing this involves showing that the data requires $D$, whether or not one assumes B. Abstract though they may be, arguments of this type are vital for an understanding of the theoretical and empirical bases of linguists' conclusions.

\section{THEORETICAL ORIENTATION}

The advent of generative grammar in the late 1950s brought an increased interest in syntax and many new results in the field. Within generative grammar, there was a period of theoretical consensus extending to the late 1960 s, at which point syntacticians began to diverge in various directions. A full understanding of the different trends in syntax in the 1970s requires an understanding of the work done in the previous decade. For this reason, the theoretical orientation of this book is very roughly that of the middle-to-late 1960 s. However, we have augmented that orientation in several respects. For example, the book does not assume rule ordering, it includes new arguments for the cycle and certain other principles, and it incorporates a significant number of analyses drawn from the 1970s. The final chapter (part 8), dealing with pronominal anaphora, is used to point the way to some of the most prominent theoretical paradigms that arose in the 1970s. 


\section{TREATMENT OF FORMALISM}

This book contains brief discussions of the formalism of transformational generative grammar. However, we do not dwell on it to any great extent. Although we do not discourage interest in formalism, we have found that an excessive emphasis on it at the beginning of one's study of syntax can get in the way of learning how to construct arguments and of assimilating the empirical basis of important results in the field. Those who wish to pursue formal issues in more detail may find it useful to consult other sources in conjunction with this book.

\section{HOW TO USE THIS BOOK}

The book consists of arguments, discussions, and a large number of problems that require the student to construct syntactic arguments. Many of the problems have discussions and sample solutions in the back of the book. In some cases the discussions are relatively complete; in others they amount only to hints for solving the problem in the text. There are also problems for which no discussion is given. A student who wants to become proficient in syntactic argumentation, and who wishes to derive full benefit from the problems, should work through each of them carefully before consulting the discussions. Problems for which complete discussions are not given can be used by teachers for assignments or springboards for class discussion.

At the end of each of the eight parts of the text is a section relating the material in that part to the broader linguistic literature. At the end of the entire book is a section called "Some Further Issues," which discusses issues and questions that may arise when working through various parts of the text. The material in this section is not a prerequisite for understanding and working through the text, but is intended for the reader who is interested in pursuing certain questions in greater detail.

This book developed out of an intensive introductory syntax course and has been classroom-tested at four universities. It assumes no previous background in linguistics and contains sufficient material for a two-semester sequence. It can also be used in intermediate or advanced syntax courses, either alone or in combination with other materials. Courses beyond the introductory level may use the early parts as review and then proceed through the rest of the book, or may use only those portions that deal with topics of particular interest.

In addition to use in courses, the book has been designed as a text for possible self-study and as a source book of important arguments and results that may be useful to both professional linguists and to scholars in other fields who wish to learn more about generative syntax. 
